\title{
Velocidade do alcance em tarefas de atenção e memória: validação do teste como marcador funcional na população geriátrica
}

\author{
Attainment speed in attention and memory tasks: test validation as a \\ functional indicator in the geriatric population
}

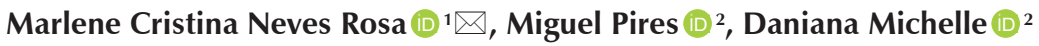 \\ 1 Polythecnic Institute from Leiria, Center for Innovative Care and Health Technology (ciTechCare). Leiria, Portugal. \\ 2 Polythecnic Institute from Leiria, School of Health Sciences, Polythecnic Institute from Leiria. Leiria, Portugal.
}

Como citar este artigo (How to cite this article):

Rosa MCN, Pires M, Michelle D. Velocidade do alcance em tarefas de atenção e memória: validação do teste como marcador funcional na população geriátrica (Attainment speed in attention and memory tasks: test validation as a functional indicator in the geriatric population). Sci Med. 2019;29(3):e34231. https://doi.org/10.15448/1980-6108.2019.3.34231

\section{RESUMO}

OBJETIVO: Validar a velocidade do alcance durante tarefas de atenção e memória como um indicador funcional na população geriátrica. MÉTODOS: Idosos institucionalizados residentes em 4 instituições no centro de Portugal foram caraterizados quanto ao seu perfil funcional, constando as seguintes variáveis: (i) cognição; (ii) independência nas atividades da básicas da vida diária (AVD); (iii) independência nas atividades instrumentais da vida diária (AIVD); (iii) força de preensão, (iv) diagnóstico de depressão; (v) função sensório-motora membro superior; (vi) funções executivas. Os vários instrumentos foram submetidos a um processo de linking com a Classificação Internacional de Funcionalidade e Saúde (CIF). A amostra foi dividida em quatro grupos funcionais considerando os valores abaixo e superiores à média da velocidade de alcance em tarefas de atenção (Grupo 1 - G1, Grupo 2 - G2) e a velocidade de alcance em tarefas de memória (Grupo 3 - G3, Grupo 4 - G4). A caraterização dos grupos de amostra foi realizada utilizando medidas de estatística descritiva dos qualificadores da CIF e a diferença entre grupos $(\mathrm{G} 1 / \mathrm{G} 2$ e G3/G4) foi testada utilizando testes t para amostras independentes.

RESULTADOS: Os resultados mostraram diferenças significativas entre grupos de baixo e elevado perfil nos testes de alcance ( $\mathrm{p}<0.05$ ), identificando as seguintes áreas prioritárias de intervenção em idosos institucionalizados: d6. Vida doméstica, d1. Aprendizagem e aplicação de conhecimento, d4. Mobilidade, d5. Autocuidados e d8. Áreas significativas da vida. Idosos com velocidade no teste de alcance em tarefas de atenção $<0.06 \mathrm{~m} / \mathrm{s}$ ou com valores $<0.07 \mathrm{~m} / \mathrm{s}$ no teste de alcance em tarefas de memória tendem a apresentar uma incapacidade moderada a grave em áreas como a Mobilidade e os Autocuidados e uma incapacidade grave a completa na participação de tarefas da Vida Doméstica CONCLUSÃO: Este estudo foi um importante contributo para a validação da velocidade do alcance (um teste para a função do membro superior) durante tarefas de atenção e memória como um indicador funcional, possibilitando a avaliação funcional de um largo espectro de idosos, incluindo os não ambulantes.

UNITERMOS: Classificação Internacional de Funcionalidade e Saúde; idoso; atenção; memória

\section{ABSTRACT}

AIM: To validate the reaching speed during attention and memory tasks as a functional indicator in the geriatric population.

METHODS: Institutionalized elderly living in 4 Portuguese institutions were characterized by their functional profile, with the following variables: (i) cognition; (ii) independence in basic daily living activities; (iii) independence in instrumental activities of daily living; (iii) grip strength, (iv) diagnosis of depression; (v) upper limb sensorimotor function; (vi) executive functions. All these instruments underwent a linking process with the International Classification of Functioning and Health. The sample was divided into four functional groups considering the values below and above the mean range of reaching speed in attention tasks (Group 1 - G1, Group 2 - G2) and the range of RS in memory tasks (Group 3 - G3, Group 4 - G4). Characterization of the sample groups was performed using descriptive statistical measures of the ICF qualifiers and the difference between groups (G1 / G2 and G3 / G4) was tested using t-tests for independent samples.

RESULTS: The results showed significant differences between low- and high-profile groups in RS tasks ( $\mathrm{p}<0.05)$, identifying the following priority intervention areas in institutionalized elderly: d6. Domestic life, d1. Learning and knowledge application, d4. Mobility, d5. Self-care and d8. Significant areas of life. Older adults with reaching speed in attention tasks $<0.06 \mathrm{~m} / \mathrm{s}$ or $<0.07 \mathrm{~m} / \mathrm{s}$ in memory tasks tend to have moderate to severe disability in Mobility and Self-Care and severe/complete inability to participate in Household tasks.

CONCLUSION: This study was an important contribution to the validation of reaching speed (a test for upper limb function) during attention and memory tasks as a functional indicator, enabling elderly functional evaluation in a large spectrum, including non-ambulate elderly people. KEYWORDS: International Classification of Functioning; Disability and Health; elderly; attention; memory. 
Abreviaturas: AA, Teste de Alcance em Tarefas de Atenção; AM, Teste de Alcance em Tarefas de Memória; AVD, Atividades Básicas da Vida Diária; AIVD, Atividades Instrumentais da Vida Diária; CIF, Classificação Internacional de Funcionalidade em Saúde; EGD, Escala Geriátrica de Depressão; FAB, Bateria de Avaliação Frontal; G1, Grupo 1; G2, Grupo 2; G3, Grupo 3; G4, Grupo 4; IB, Índice de Barthel; ILB, Indice de Lawton \& Brody; MEEM, Mini Exame De Estado Mental.

\section{INTRODUÇÃO}

O processo de envelhecimento saudável é definido como um "processo de otimização das oportunidades de saúde, participação social e segurança, de forma a promover a qualidade de vida ao longo do envelhecimento" [1]. Ao conceito de envelhecimento saudável está intimamente ligado o conceito de capacidade funcional, definido pela manutenção da autonomia e independência do idoso na sua vida diária [1]. Para o planeamento de intervenções específicas que visem a minimização da incapacidade funcional da população idosa, é crucial validar indicadores de capacidade funcional [2]. Assim, tem sido consensual na investigação atual sobre o tópico do envelhecimento $[3,4]$ que a avaliação do perfil funcional é um elemento essencial na gestão dos cuidados da pessoa idosa.

Vários indicadores têm sido usados para determinar e caracterizar este perfil funcional, tais como testes de função cognitiva - Mini Exame de Estado Mental (MEEM) [5], testes de independência nas Atividades da Vida Diária (AVD) - Índice de Barthel (IB) [6], Índice Lawton \& Brody (ILB) [7], Índice de Katz [8], testes de preensão manual $[9,10]$, testes de equilíbrio Escala de Berg [11], Balance Scale [12], questionários de Avaliação Funcional e Multidimensional para Idosos [13], testes de força/velocidade do membro inferior [14], testes de marcha: 10-Meter Walk Test, 6 Minute Walk Test; testes de equilíbrio na marcha: Dynamic Gait Index [15], Timed-Up and Go Test [8]. Os testes de marcha são frequentemente a escolha dos profissionais de saúde e dos investigadores para, de forma ágil e válida, avaliar o perfil funcional do idoso. Assim, com exceção do teste de preensão manual $[9,10]$, que tem demonstrado algumas limitações por não ser sensível à identificação de restrições em idosos com diminuição da função executiva ou cognitiva [16], poucos indicadores funcionais existem adaptados a pessoas com inabilidade de marcha $[17,18]$. Com este enquadramento, Rosa et al. desenvolveram o teste da velocidade do alcance em tarefas de atenção e em tarefas de memória, visando constituir um instrumento para avaliar, monitorizar e reabilitar a função cognitivo-motora dos membros superiores na pessoa idosa com restrições da funcionalidade [19]. O teste tem demonstrado correlações significativas entre as suas pontuações e a habilidade sensório-motor no idoso, mas carece de estudos mais robustos quanto à sua habilidade para detetar um perfil funcional específico [20].

Assim, considerando o potencial deste teste na avaliação funcional do idoso em conjunto com a tendência na escolha de indicadores funcionais de marcha e tendo em conta as opções reduzidas e limitadas de indicadores baseados na função dos membros superiores - especialmente importantes para o idoso com limitação ou incapacidade de locomoção -, [21] este estudo propõe-se a validar a velocidade do alcance (durante tarefas de atenção e memória) como um indicador funcional na população geriátrica.

\section{MÉTODOS}

Um estudo descritivo foi realizado em quatro instituições do Consórcio da Rede Social da região Centro de Portugal: Unidade de Convalescença, Santa Casa da Misericórdia do Entroncamento; Lar da Associação Social de Avelãs de Caminho, Lar da Santa Casa da Misericórdia de Pombal, Lar do Centro Social e Recreativo da Pedralva. O estudo recebeu aprovação ética em reunião de consórcio a 20 de março de 2019. Os participantes potencialmente elegíveis foram identificados e abordados pelo diretor técnico de cada instituição, sendo que os que mostraram interesse em participar foram contactados pelo investigador e foi agendada a avaliação. Foi fornecida informação escrita aos participantes e os mesmos assinaram um termo de consentimento livre e esclarecido. O presente estudo adotou como critério de inclusão, pessoas com idade igual ou superior a 60 anos, não acamadas e capazes de compreender pelo menos dois comandos verbais simples. Foram excluídos idosos em estados graves de alterações de consciência ou com afasia global ou de compreensão e com limitações severas de visão.

A coleta de dados ocorreu nos meses de março e abril de 2019. O perfil funcional foi obtido através de um conjunto de instrumentos de avaliação para caracterização geral da amostra e caracterização do perfil funcional. A coleta foi realizada por dois investigadores treinados, ficando cada um responsável por duas instituições parceiras. 
Foi implementado um questionário sociodemográfico e clínico que demorou cerca de 20 minutos para cada participante, estando dividido em oito principais itens: nome, gênero, idade, escolaridade (em anos estudados), situação laboral (empregado, desempregado ou reformado), estado civil, tipo de suporte social formal (centro dia, convívio, fisioterapia em instituição, internamento, unidade de cuidados continuados ou residente em lar) e a presença de diagnóstico clínico de doença.

Foram consideradas sete variáveis para a avaliação funcional do idoso, recolhidas com os seguintes instrumentos de avaliação: (i) a força de preensão manual ( $\mathrm{kg} /$ força), que foi aferida com o dinamômetro K-Force Grip ${ }^{\circledR}$ (Kinvent, Orsay, França), em ambas as mãos. O procedimento de recolha consistiu em: o idoso na posição de sentado, com os pés apoiados ao solo e o membro superior a ser testado mantido com o cotovelo em flexão de $90^{\circ}$, ombro em adução e antebraço na posição neutra. Para cada idoso, foram realizadas três preensões máximas, com duração de cinco segundos, intercaladas e foi registada a melhor performance. Foram consultados os valores normativos ajustados ao gênero e à idade de acordo Adedoyin et al. [22]; (ii) o MEEM foi aplicado para o screening do estado cognitivo da população geriátrica em estudo. Este teste caracteriza-se por duas partes distintas: uma primeira parte que abrange orientação, memória e atenção, com pontuação máxima de 21 pontos; uma segunda parte que aborda habilidades específicas como nomear e compreender, com pontuação máxima de nove pontos, totalizando um score de 30 pontos [23]. A pontuação do teste foi corrigida de acordo com a escolaridade: mais de 11 anos de escolaridade considera-se a função cognitiva afetada quando a pontuação é $\leq 27$; entre 1 e 11 anos de escolaridade considera-se que a cognição está afetada quando $\leq 22$ pontos; e pessoa sem escolaridade, a cognição está afetada se a pontuação for $\leq 9$ [24]; (iii) a Escala Geriátrica de Depressão (EGD) foi utilizada para avaliar o estado de depressão, abordando aspetos cognitivos e comportamentais tipicamente afetados na depressão do idoso. A informação é obtida através de questionário direto ao idoso, com 30 questões com resposta dicotômica (sim ou não). As respostas sugestivas de existência de depressão correspondem a um ponto, os valores de 0 a 10 pontos são indicadores de ausência de depressão, 11 a 20 pontos de depressão ligeira e de 21 a 30 pontos, de depressão grave [25]; (iv) o IB - é um instrumento que avalia o nível de independência em dez atividades básicas de vida diária: comer, higiene pessoal, uso do toilet, tomar banho, vestir e despir, controle de esfíncteres, deambular, transferência da cadeira para a cama, subir e descer escadas. A pontuação da escala varia de 0 a 100 , sendo a pontuação mínima de zero correspondente a máxima dependência para todas as AVD avaliadas, e a pontuação máxima de 100 equivalente a independência total [26]; (v) a Bateria de Avaliação Frontal (FAB) - é uma bateria que consiste em seis subtestes que exploram: conceptualização, flexibilidade mental, programação motora, sensibilidade à interferência, controlo inibitório e autonomia ambiental. Cada subteste é classificado de zero a três pontos, conforme o desempenho do usuário, sendo que quanto maior a pontuação, mais preservada estará a função executiva [27]; (vi) a Escala de Avaliação Fugl-Meyer, que está dividida em cinco domínios: função motora, sensibilidade, equilíbrio, amplitude de movimento e dor [28]. Neste estudo, foi aplicada a sua versão para membro superior. Esta versão da escala está dividida em quatro categorias (extremidade superior, mão, punho e coordenação/velocidade), variando o score total de 0 a 66 , onde 66 representa o máximo de função motora do membro superior; (vii) o ILB, que avalia o desempenho nas atividades instrumentais da vida diária, considerando oito tarefas diferentes: utilização do telefone, realização de compras, preparação das refeições, tarefas domésticas, lavagem da roupa, utilização de meios de transporte, gestão da medicação e responsabilidade de assuntos financeiros. O score final varia entre zero e oito pontos, onde o escore de oito pontos corresponde à maior independência [29].

Com base nestes instrumentos de avaliação, foi realizado o linking com a Classificação Internacional de Funcionalidade, Incapacidade e Saúde (CIF). A CIF é um instrumento reconhecido internacionalmente para a descrição funcional em saúde, recomendada na avaliação de populações de risco, inclusive na população geriátrica [30]. Considerando a sua extensão e detalhe, as regras de linking foram desenvolvidas para permitir a transposição de outros instrumentos e metodologias para a linguagem standard e universal da CIF [31]. Assim, neste estudo, os vários instrumentos foram submetidos a um processo de linking para caracterização do perfil funcional da amostra [31,32]. O processo de linking foi assegurado por dois investigadores. A ligação entre as categorias da CIF identificadas e os itens de cada instrumento foi feita por um investigador e revista pelo investigador mais experiente, seguindo um procedimento específico para cada variável e descrito na Tabela 1 [23-27]. 
Tabela 1. Descrição do processo de linking dos vários instrumentos de avaliação (Força de preensão manual, Mini-Exame de Estado Mental, Escala Geriátrica de Depressão, índice de Barthel, Bateria de Avaliação Frontal, Escala Fugl-Meyer, Índice de Lawton e Brody) com a Classificação Internacional de Funcionalidade, Incapacidade e Saúde.

\begin{tabular}{|c|c|c|c|c|c|}
\hline \multirow{2}{*}{$\begin{array}{l}\text { Instrumento } \\
\text { K-force }\end{array}$} & \multirow{2}{*}{$\begin{array}{l}\text { Item } \\
\text { Força de Preensão Manual (kg/f) }\end{array}$} & \multirow{2}{*}{$\begin{array}{l}\text { Categoria } \\
\text { b7300 - Força de músculos isolados e } \\
\text { grupos musculares (em kgf) }\end{array}$} & \multicolumn{2}{|c|}{ Escore } & \multirow[b]{2}{*}{$\begin{array}{c}\text { CIF } \\
\\
0 \\
1 \\
2 \\
3 \\
4\end{array}$} \\
\hline & & & $\begin{array}{c}\text { Membro direito } \\
23.04-30.70 \\
15.36-23.03 \\
7.69-15.35 \\
0.01-7.68 \\
0.00\end{array}$ & $\begin{array}{c}\text { Membro esquerdo } \\
24.54-32.70 \\
16.36-24.53 \\
8.19-16.35 \\
0.01-8.18 \\
0.00\end{array}$ & \\
\hline \multirow[t]{5}{*}{ MEEM } & Orientação & b114 - Orientação & \multicolumn{2}{|c|}{$\begin{array}{c}9-10 / 10 \\
7-8 / 10 \\
5-6 / 10 \\
3-4 / 10 \\
0-3 / 10\end{array}$} & $\begin{array}{l}0 \\
1 \\
2 \\
3 \\
4\end{array}$ \\
\hline & Atenção e Cálculo & b1400 - Manutenção da atenção & \multicolumn{2}{|c|}{$\begin{array}{c}5 / 5 \\
4 / 5 \\
3 / 5 \\
2 / 5 \\
1 / 5 \text { ou } 0 / 5\end{array}$} & $\begin{array}{l}0 \\
1 \\
2 \\
3 \\
4\end{array}$ \\
\hline & Retenção, Evocação & $\begin{array}{l}\text { b1440 - Memória de curto prazo } \\
\text { b1442 - Recuperação da memória }\end{array}$ & $\begin{array}{c}\text { Retenção } \\
3 / 3 \\
2 / 3 \\
1 / 3 \\
\geq 1 / 3 \\
0 / 3\end{array}$ & $\begin{array}{c}\text { Evocação } \\
3 / 3 \\
2 / 3 \\
1 / 3 \\
\geq 1 / 3 \\
0 / 3\end{array}$ & $\begin{array}{l}0 \\
1 \\
2 \\
3 \\
4\end{array}$ \\
\hline & Linguagem (alíneas a), b), d) e e)) & b167 - Funções mentais da linguagem & $1 / 5$ & $\begin{array}{l}15 \\
15 \\
15 \\
15 \\
1 \\
0 / 5\end{array}$ & $\begin{array}{l}0 \\
1 \\
2 \\
3 \\
4\end{array}$ \\
\hline & $\begin{array}{l}\text { Linguagem (alínea c)), Habilidade } \\
\text { Construtiva }\end{array}$ & d210 - Realizar uma única tarefa & & $\begin{array}{l}14 \\
14 \\
14 \\
14 \\
14\end{array}$ & $\begin{array}{l}0 \\
1 \\
2 \\
3 \\
4\end{array}$ \\
\hline EGD & Total items (30 questões) & b152 - Funções emocionais & & $\begin{array}{l}0 / 30 \\
0 / 30 \\
0 / 30 \\
30\end{array}$ & $\begin{array}{l}0 \\
1 \\
3 \\
4\end{array}$ \\
\hline \multirow[t]{10}{*}{ IB } & Função do Intestino & b525 - Funções de defecação & & $\begin{array}{l}10 \\
10 \\
10\end{array}$ & $\begin{array}{l}0 \\
2 \\
4\end{array}$ \\
\hline & Função Sistema Urinário & b620 - Funções miccionais & & $\begin{array}{l}10 \\
10 \\
10\end{array}$ & $\begin{array}{l}0 \\
2 \\
4\end{array}$ \\
\hline & $\begin{array}{l}\text { Transferência (da cama para a cadeira e } \\
\text { vice-versa) }\end{array}$ & d420 - Autotransferências & & $\begin{array}{l}15 \\
15 \\
15 \\
15\end{array}$ & $\begin{array}{l}0 \\
1 \\
3 \\
4\end{array}$ \\
\hline & Mobilidade & $\begin{array}{l}\text { d450 - Andar } \\
\text { d455 - Deslocar-se } \\
\text { d465 - Deslocar-se utilizando algum } \\
\text { tipo de equipamento }\end{array}$ & & $\begin{array}{l}15 \\
15 \\
15 \\
15\end{array}$ & $\begin{array}{l}0 \\
1 \\
3 \\
4\end{array}$ \\
\hline & Escadas & d4553 - Subir/descer & & $\begin{array}{l}10 \\
10 \\
10\end{array}$ & $\begin{array}{l}0 \\
2 \\
4\end{array}$ \\
\hline & Banho & d510 - Lavar-se & & $\begin{array}{l}5 \\
/ 5\end{array}$ & $\begin{array}{l}0 \\
4\end{array}$ \\
\hline & Atividades Rotineiras & d520 - Cuidar de partes do corpo & & $\begin{array}{l}/ 5 \\
/ 5\end{array}$ & $\begin{array}{l}0 \\
4\end{array}$ \\
\hline & Uso do Toilet & $\begin{array}{l}\text { d530 - Cuidados relacionados com os } \\
\text { processos de excreção }\end{array}$ & & $\begin{array}{l}10 \\
10 \\
10\end{array}$ & $\begin{array}{l}0 \\
2 \\
4\end{array}$ \\
\hline & Vestir-se & d540 - Vestir-se & & $\begin{array}{l}10 \\
10 \\
10\end{array}$ & $\begin{array}{l}0 \\
2 \\
4\end{array}$ \\
\hline & Alimentação & d550 - Comer & & $\begin{array}{l}10 \\
10 \\
10\end{array}$ & $\begin{array}{l}0 \\
2 \\
4\end{array}$ \\
\hline
\end{tabular}


Tabela 1 (continuação)

\begin{tabular}{|c|c|c|c|c|}
\hline Instrumento & Item & Categoria & Escore & CIF \\
\hline \multirow[t]{2}{*}{ FAB } & $\begin{array}{l}\text { Concetualização, Flexibilidade } \\
\text { Mental, Programação, Sensibilidade } \\
\text { à Interferência, Controlo Inibitório, } \\
\text { Autonomia Ambiental }\end{array}$ & $\begin{array}{l}\text { b164 - Funções cognitivas de nível } \\
\text { superior } \\
\text { d163 - Pensar }\end{array}$ & $\begin{array}{c}18 / 18 \\
13-17 / 18 \\
6-12 / 18 \\
1-5 / 18 \\
0 / 18\end{array}$ & $\begin{array}{l}0 \\
1 \\
2 \\
3 \\
4\end{array}$ \\
\hline & $\begin{array}{l}\text { Programação, Sensibilidade à } \\
\text { Interferência, Controlo Inibitório }\end{array}$ & d220 - Realizar tarefas múltiplas & $\begin{array}{c}9 / 18 \\
6-8 / 18 \\
4-5 / 18 \\
1-3 / 18 \\
0 / 18\end{array}$ & $\begin{array}{l}0 \\
1 \\
2 \\
3 \\
4\end{array}$ \\
\hline \multirow[t]{4}{*}{$\begin{array}{l}\text { Escala Fugl-Meyer } \\
\text { (sub-score do } \\
\text { membro superior) }\end{array}$} & Extremidade Superior, Punho, Mão & b730 - Funções da força muscular & $\begin{array}{c}60 / 60 \\
40-59 / 60 \\
21-39 / 60 \\
1-20 / 60 \\
0 / 60\end{array}$ & $\begin{array}{l}0 \\
1 \\
2 \\
3 \\
4\end{array}$ \\
\hline & Extremidade Superior & $\begin{array}{l}\text { s720 - Estrutura da região do ombro } \\
\text { s73001 - Articulações da região do } \\
\text { cotovelo } \\
\text { s73002 - Músculos do braço } \\
\text { s7301 - Estrutura do antebraço }\end{array}$ & $\begin{array}{c}36 / 36 \\
24-35 / 36 \\
13-23 / 36 \\
1-12 / 36 \\
0 / 36\end{array}$ & $\begin{array}{l}0 \\
1 \\
2 \\
3 \\
4\end{array}$ \\
\hline & Punho & s73011 - Articulações do punho & $\begin{array}{c}10 / 10 \\
7-9 / 10 \\
4-6 / 10 \\
1-3 / 10 \\
0 / 10\end{array}$ & $\begin{array}{l}0 \\
1 \\
2 \\
3 \\
4\end{array}$ \\
\hline & Mão & $\begin{array}{l}\text { s7302 - Estrutura da mão } \\
\text { s73022 - Músculos da mão } \\
\text { d4401 - Agarrar }\end{array}$ & $\begin{array}{c}14 / 14 \\
9-13 / 14 \\
6-8 / 14 \\
1-5 / 14 \\
0 / 14\end{array}$ & $\begin{array}{l}0 \\
1 \\
2 \\
3 \\
4\end{array}$ \\
\hline \multirow[t]{8}{*}{ ILB } & Capacidade para usar o telefone & $\begin{array}{l}\text { d360 - Utilização de dispositivos e de } \\
\text { técnicas de comunicação }\end{array}$ & $\begin{array}{l}1 / 1 \\
0 / 1\end{array}$ & $\begin{array}{l}0 \\
4\end{array}$ \\
\hline & Compras & d6200 - Comprar & $\begin{array}{l}1 / 1 \\
0 / 1\end{array}$ & $\begin{array}{l}0 \\
4\end{array}$ \\
\hline & Preparação das refeições & d630 - Preparar refeições & $\begin{array}{l}1 / 1 \\
0 / 1\end{array}$ & $\begin{array}{l}0 \\
4\end{array}$ \\
\hline & Cuidar da casa & d640 - Realizar as tarefas domésticas & $\begin{array}{l}1 / 1 \\
0 / 1\end{array}$ & $\begin{array}{l}0 \\
4\end{array}$ \\
\hline & Lavagem da roupa & d6400 - Lavar e secar roupa & $\begin{array}{l}1 / 1 \\
0 / 1\end{array}$ & $\begin{array}{l}0 \\
4\end{array}$ \\
\hline & Deslocações & d470 - Utilização de transporte & $\begin{array}{l}1 / 1 \\
0 / 1\end{array}$ & $\begin{array}{l}0 \\
4\end{array}$ \\
\hline & Responsabilidade sobre a medicação & d5702 - Manter a própria saúde & $\begin{array}{l}1 / 1 \\
0 / 1\end{array}$ & $\begin{array}{l}0 \\
4\end{array}$ \\
\hline & Capacidade para usar o dinheiro & d860 - Transações económicas básicas & $\begin{array}{l}1 / 1 \\
0 / 1\end{array}$ & $\begin{array}{l}0 \\
4\end{array}$ \\
\hline
\end{tabular}

CIF, Classificação Internacional de Funcionalidade; EGD: Escala Geriátrica de depressão; kgf: kilograma/força; MEEM: Mini-Exame de Estado Mental.

Para avaliação da velocidade do alcance durantes tarefas de atenção e durante tarefas de memória, foi implementado o protocolo Agilidades. Este protocolo dispõe de um teste de Alcance para Tarefas de Atenção (AA) e de um teste de Alcance para Tarefas de Memória (AM), ambos desenvolvidos no Instituto Politécnico de Leiria. A realização destes testes requer um conjunto sistematizado de movimentos, especialmente adaptadas às possibilidades e às necessidades das pessoas idosas. O instrumento de avaliação, consiste num tabuleiro com 24 figuras geométricas com formas e cores diferentes, cada uma com $12 \mathrm{~cm}$ por $14 \mathrm{~cm}$ de dimensão, distribuídas em quatro linhas e seis colunas, dispostas lado a lado. A disposição destas figuras é separada por uma linha média que dispõe de forma simétrica três colunas para o lado direito e três para o esquerdo. Entre a terceira e quarta linhas e a quinta e sexta coluna, foram colocadas mais duas figuras do lado direito e duas do lado esquerdo, contabilizando um total de 30 figuras. As figuras foram distribuídas de forma em que a distância da base de início do teste a cada uma das figuras pudesse ser aferida (Figura 1). 


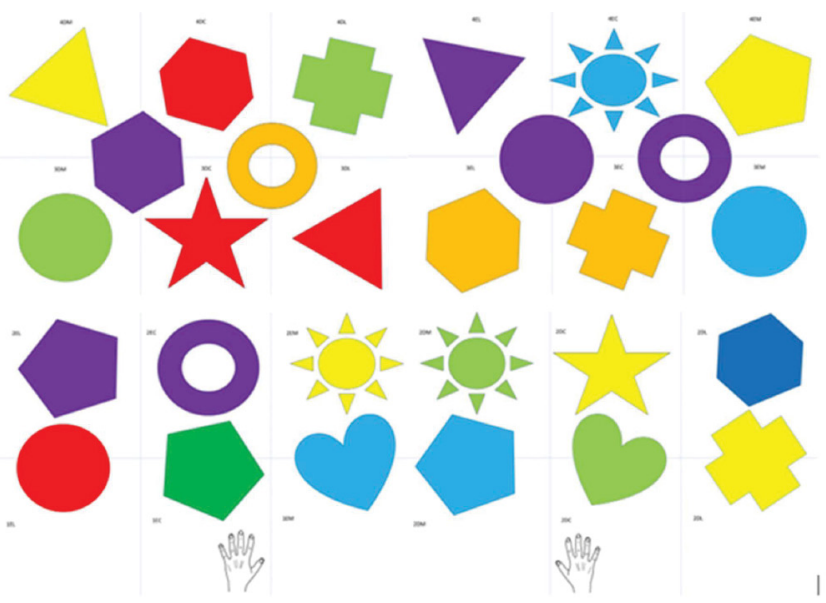

Figura 1. Apresentação do tabuleiro para o desempenho dos testes de alcance em tarefas de atenção e em tarefas de memória.

Este tabuleiro desenhado para o teste do alcance tem a flexibilidade de facultar dois testes de tarefas diferentes, nomeadamente as tarefas de atenção e memória. $\mathrm{O}$ teste do AA consta de 10 cartas, de figuras geométricas com formas e cores diferentes. Cada carta tem uma informação adicional sugerindo qual o membro que deverá realizar o alcance, podendo constar a opção de alcançar a figura com a mão direita ou com a mão esquerda. A distância final das figuras alcançadas neste teste é de 8.9 metros. O teste do AM utiliza o mesmo tabuleiro, mas com cartas que apresentam desafios diferentes. Este teste é constituído por nove cartas, formando o conjunto de três pares de figuras. Depois de alcançado cada par de cartas, é solicitado aos idosos que repita o alcance realizado nas duas últimas cartas através do processo de memorização e retenção motora e visual. A contabilização total das nove cartas, mais as suas repetições, resulta numa distância total de 12.4 metros. Para a realização do teste, o tabuleiro é colocado numa mesa de altura ajustada a cada participante, a uma distância de $5 \mathrm{~cm}$ da face anterior do tronco do examinado; esse espaço foi necessário para que cada participante pudesse pousar a mão direita e esquerda sobre a mesa, em dois locais indicados na base do tabuleiro. O teste somente dá início quando os participantes estão sentados e com as mãos posicionadas adequadamente e com o tronco em posição central ao tabuleiro. As instruções a respeito do objetivo do teste são dadas a cada participante e só após o entendimento das regras o examinador inicia cronometragem do tempo, mostrando imediatamente a primeira carta com a figura a ser alcançada. $\mathrm{O}$ instrumento de avaliação AA e AM foram aplicados em cada participante, o tempo total do teste foi cronometrado e a distância percorrida (previamente conhecida) foi usada para cálculo da velocidade. Os participantes que apresentaram cinco ou mais erros durante a aplicação dos testes foram considerados sem critério para realização e a sua performance foi considerada nula.

Após a coleta dos dados, foi realizada a análise estatística. Procedeu-se à divisão da amostra em estudo em dois grupos funcionais, considerando a velocidade de alcance em tarefas de atenção: Grupo 1 (G1), a população que completou o teste com valores superiores à média de $0.06 \mathrm{~m} / \mathrm{s}$ foi classificada com baixo perfil de velocidade de alcance; Grupo 2 (G2), a população que completou o teste com valores inferiores à média foi considerada como elevado perfil de velocidade de alcance. Em complemento, a amostra foi divida em dois grupos funcionais considerando a velocidade de alcance em de tarefas de memória: Grupo 3 (G3), a população com desempenho no teste com valores superiores à média de $0.07 \mathrm{~m} / \mathrm{s}$ foi classificada com baixo perfil de velocidade de alcance com memória, e Grupo 4 (G4), a população que teve um desempenho inferior à média e foi classificada com elevado perfil de velocidade de alcance.

Para cada um dos grupos de amostra, foi realizada uma caracterização dos dados sociodemográficos, clínica e funcional utilizando medidas de estatística descritiva dos qualificadores da CIF atribuídos (médias, desvio padrão e frequências). Para avaliar as diferenças estatísticas entre os valores médios dos qualificadores entre os grupos foram aplicados os testes t-student para amostras independentes. Os dados da amostra foram analisados através do IBM SPSS Statistics 25.0. O valor de $\mathrm{p}$ foi considerado estatisticamente significativo quando menor que 0,05 .

\section{RESULTADOS}

No total, foram avaliados 106 idosos, não tendo sido nenhum excluído. Os dados demonstram que a amostra deste estudo é maioritariamente feminina em todos os grupos. A Idade média da amostra encontrase acima dos 75 anos em todos os grupos com valores ligeiramente mais baixos nos grupos $\mathrm{G} 2(77,3 \pm 11,2)$ e G4 $(78,3 \pm 11,2)$. Importante referir que os utentes da amostra representam uma escolaridade média maior nos grupos com elevado perfil $(\mathrm{G} 2=3,9 \pm 3,5$ anos; $\mathrm{G} 4=3,9 \pm 3,7$ anos). A situação laboral da amostra revela que a esmagadora maioria da população (>95\%) é aposentada, não havendo grandes variações entre os mesmos. Mais de metade da amostra apresenta estado civil de viuvez (Tabela 2). As pessoas com pior desempenho nos testes de atenção e memória 
foram as que compuseram a grande parte da população institucionalizada em lar (G1-52,0\%; G3-47,4,\%), assim como nas unidades de cuidados continuados (G112,0\%; G3-15,8\%). As pessoas com melhor desempenho nestes testes estão, em grande parte, nos centros de dia (G2-39,6,\%; G4-39,1\%) e a frequentar fisioterapia (G29,4\%; G4-10,4\%). Em termos de participantes com diagnóstico, a maior parte dos que tinham condição médica definida foram enquadrados nos grupos G1 (74,0\%) e G3 (73,7\%), havendo uma redução destas percentagens nos grupos de elevado perfil.

De uma forma global, conforme observado na Tabela 3, os grupos de elevado perfil para a velocidade de alcance (G2 e G4) evidenciaram resultados mais elevados nas diferentes variáveis funcionais: cognição (MEEM); funções executivas (FAB); independência nas AVD e nas atividades instrumentais da vida diária; função sensório motora do membro superior e força de preensão manual para ambos os membros direito e esquerdo, respetivamente. Em exceção a esta tendência demonstrada pelos nossos resultados, a EGD apresenta uma tendência inversa, com resultados mais elevados para os grupos de baixo perfil para a velocidade de alcance.
Os grupos de baixo perfil de velocidade de alcance (G1 e G3) apresentaram valores entre $0.03 \pm 0,02 \mathrm{~m} / \mathrm{s}$ no teste de AA e $0.03 \pm 0,02 \mathrm{~m} / \mathrm{s}$ no teste de AM. Os grupos de elevado perfil de velocidade de alcance (G3 e G4) apresentaram valores entre $0,09 \pm 0,02 \mathrm{~m} / \mathrm{s}$ no AA e $0,14 \pm 0,052 \mathrm{~m} / \mathrm{s}$ no AM.

De todas as categorias identificadas, apenas as categorias b152 (funções emocionais), b525 (funções de defecação), d550 (comer) e d6400 (lavar e secar roupa) não mostraram diferenças significativas entre os grupos de baixo e elevado perfil de alcance em tarefas de atenção. Considerando os valores de velocidade do alcance em tarefas de memória as categorias que estatisticamente não apresentaram diferenças significativas foram: b152 (funções emocionais), b5 (funções do aparelho digestivo e dos sistemas metabólico e endócrino), b525 (funções de defecação), b6 (funções geniturinárias e reprodutivas) e b620 (funções miccionais). As categorias de b1440 (memória de curto prazo), b1442 (recuperação da memória) apresentaram uma tendência para a significância estatística $(\mathrm{p}=0.05)$ quando comparados os grupos de baixo e elevado perfil de alcance em tarefas de memória (Tabela 4).

Tabela 2. Tabela de caracterização sociodemográfica da amostra.

\begin{tabular}{|c|c|c|c|c|}
\hline & G1 & G2 & G3 & G4 \\
\hline $\mathrm{n}$ & 52 & 54 & 58 & 48 \\
\hline Sexo feminino (\%) & 70.0 & 60.4 & 70.2 & 58.7 \\
\hline Idade, (anos), média \pm desvio-padrão & $80.2 \pm 9.2$ & $77.3 \pm 11.2$ & $79.0 \pm 9.7$ & $78.3 \pm 11.2$ \\
\hline Escolaridade, (anos), média \pm desvio-padrão & $2.4 \pm 2.7$ & $3.9 \pm 3.5$ & $2.6 \pm 2.6$ & $3.9 \pm 3.7$ \\
\hline \multicolumn{5}{|l|}{ Situação laboral (\%) } \\
\hline Empregado & 0.0 & 3.8 & 1.8 & 2.2 \\
\hline Desempregado & 2.0 & 0.0 & 1.8 & 0.0 \\
\hline Reformado & 96.0 & 96.2 & 94.7 & 97.8 \\
\hline Não aplicáveis & 2.0 & 0.0 & 1.8 & 0.0 \\
\hline \multicolumn{5}{|l|}{ Estado civil (\%) } \\
\hline Casado & 34.0 & 22.6 & 33.3 & 21.7 \\
\hline Divorciado & 0.0 & 3.8 & 0.0 & 4.3 \\
\hline Solteiro & 10.0 & 20.8 & 15.8 & 15.2 \\
\hline Viúvo & 56.0 & 52.8 & 50.9 & 58.7 \\
\hline \multicolumn{5}{|l|}{ Suporte social formal (\%) } \\
\hline Centro de dia & 32.0 & 39.6 & 33.3 & 39.1 \\
\hline Convívio & 0.0 & 1.9 & 0.0 & 2.2 \\
\hline Fisioterapia em instituição & 4.0 & 9.4 & 3.5 & 10.9 \\
\hline Internamento & 0.0 & 1.9 & 0.0 & 2.2 \\
\hline Reside em Lar & 52.0 & 37.7 & 47.4 & 41.3 \\
\hline Unidade de Cuidados Continuados & 12.0 & 9.4 & 15.8 & 4.3 \\
\hline \multicolumn{5}{|l|}{ Diagnóstico (\%) } \\
\hline Sim & 74.0 & 64.2 & 73.7 & 63.0 \\
\hline Não & 24.0 & 35.8 & 24.6 & 37.0 \\
\hline Não Aplicável & 2.0 & 0.0 & 1.8 & 0.0 \\
\hline
\end{tabular}

G1: grupo 1 - baixo perfil na velocidade de alcance tarefas de atenção; G2: grupo 2 - baixo perfil na velocidade de alcance tarefas de atenção; G3: grupo 3 - baixo perfil na velocidade de alcance tarefas de memória; G4: grupo 4 - alto perfil na velocidade de alcance tarefas de memória. 
Tabela 3. Tabela de caraterização funcional da amostra.

\begin{tabular}{|c|c|c|c|c|c|c|}
\hline & G1 & G2 & $\mathbf{p}$ & G3 & G4 & p \\
\hline$n$ & 52 & 54 & & 58 & 48 & \\
\hline MEEM (Escore total: 0/30) & $18,8 \pm 6.1$ & $24,7 \pm 4,2$ & 0,00 & $19,4 \pm 6,0$ & $24,8 \pm 4,2$ & 0,01 \\
\hline FAB (Escore total: 0/18) & $7,4 \pm 3,9$ & $11,7 \pm 3,6$ & 0,00 & $7,7 \pm 3,9$ & $12,0 \pm 3,5$ & 0,00 \\
\hline EGD (Escore total: 0/30) & $12,7 \pm 5,6$ & $11,2 \pm 5,6$ & 0,05 & $12,7 \pm 5,6$ & $11,0 \pm 5,6$ & 0,07 \\
\hline IB (Escore total: 0/100) & $58,3 \pm 29,9$ & $80,1 \pm 24,0$ & 0,00 & $58,6 \pm 31,7$ & $83,0 \pm 18,7$ & 0,00 \\
\hline FM (Escore total: 0/126) & $94,2 \pm 28,3$ & $113,5 \pm 17,0$ & 0,00 & $95,6 \pm 28,8$ & $114,8 \pm 13,5$ & 0,00 \\
\hline ILB (Escore total: 0/8) & $2,2 \pm 2,0$ & $4,2 \pm 2,6$ & 0,01 & $2,2 \pm 0,27$ & $4,4 \pm 0,4$ & 0,01 \\
\hline \multicolumn{7}{|c|}{ Força de preensão manual (kgf) } \\
\hline MSD & $10,1 \pm 5,2$ & $12,9 \pm 6,2$ & 0,10 & $10,6 \pm 5,7$ & $12,7 \pm 6,0$ & 0,10 \\
\hline MSE & $9,7 \pm 5,5$ & $12,8 \pm 6,5$ & 0,02 & $9,4 \pm 5,5$ & $13,6 \pm 6,3$ & 0,00 \\
\hline
\end{tabular}

EGD, Escala de Depressão Geriátrica; FAB, Bateria de Avaliação Frontal; G1, grupo 1 - baixo perfil na velocidade de alcance tarefas de atenção; G2, grupo 2 - baixo perfil na velocidade de alcance tarefas de atenção; G3, grupo 3 - baixo perfil na velocidade de alcance tarefas de memória; G4, grupo 4 - alto perfil na velocidade de alcance tarefas de memória; IB, Índice de Barthel; MEEM, Mini-Exame de Estado Mental; ILB, Índice Lawton \& Brody; kgf, quilograma-força exercidos durante a força de preensão manual.

Tabela 4. Média de cada categoria Classificação Internacional de Funcionalidade nos vários tipos de perfil funcional.

\begin{tabular}{|c|c|c|c|c|c|c|}
\hline & (G1) & (G2) & $\mathbf{p}$ & (G3) & (G4) & p \\
\hline$n$ & 52 & 54 & & 58 & 48 & \\
\hline $\begin{array}{l}\text { b1 - Funções mentais } \\
\text { b114 - Funções da orientação } \\
\text { b1400 - Manutenção da atenção } \\
\text { b1440 - Memória de curto prazo } \\
\text { b1442 - Recuperação da memória } \\
\text { b152 - Funções emocionais } \\
\text { b164 - Funções cognitivas de nível superior } \\
\text { b167 - Funções mentais da linguagem }\end{array}$ & $\begin{array}{l}1,8 \pm 0,7 \\
1,5 \pm 1,3 \\
2,3 \pm 1,8 \\
2,1 \pm 1,2 \\
2,1 \pm 1,2 \\
0,8 \pm 0,9 \\
2,2 \pm 0,7 \\
1,2 \pm 0,8\end{array}$ & $\begin{array}{l}1,1 \pm 0,7 \\
0,5 \pm 0,8 \\
1,2 \pm 1,6 \\
1,6 \pm 1,4 \\
1,6 \pm 1,4 \\
0,7 \pm 0,8 \\
1,6 \pm 0,6 \\
0,6 \pm 0,7\end{array}$ & $\begin{array}{l}0,0^{*} \\
0,0^{*} \\
0,0^{*} \\
0,0^{*} \\
0,0^{*} \\
0,4 \\
0,0^{*} \\
0,0^{*}\end{array}$ & $\begin{array}{l}1,7 \pm 0,7 \\
1,4 \pm 1,3 \\
2,4 \pm 1,8 \\
2,1 \pm 1,2 \\
2,1 \pm 1,2 \\
0,8 \pm 0,8 \\
2,2 \pm 0,7 \\
1,2 \pm 0,8\end{array}$ & $\begin{array}{l}1,1 \pm 0,7 \\
0,5 \pm 0,8 \\
1,1 \pm 1,6 \\
1,6 \pm 1,4 \\
1,6 \pm 1,4 \\
0,6 \pm 0,9 \\
1,5 \pm 0,6 \\
0,6 \pm 0,7\end{array}$ & $\begin{array}{r}0,0^{*} \\
0,0^{*} \\
0,0^{*} \\
0,1 \\
0,1 \\
0,3 \\
0,0^{*} \\
0,0^{*}\end{array}$ \\
\hline $\begin{array}{l}\text { b5 - Funções do aparelho digestivo e dos sistemas metabólico endócrino } \\
\text { b525 - Funções de defecação }\end{array}$ & $0,9 \pm 1,5$ & $0,6 \pm 1,1$ & 0,2 & $0,9 \pm 1,4$ & $0,5 \pm 1,1$ & 0,1 \\
\hline $\begin{array}{l}\text { b6 - Funções geniturinárias e reprodutivas } \\
\text { b620 - Funções miccionais }\end{array}$ & $1,4 \pm 1,5$ & $0,8 \pm 1,3$ & $0,0^{*}$ & $1,3 \pm 1,5$ & $0,8 \pm 1,3$ & 0,1 \\
\hline $\begin{array}{l}\text { b7 - Funções neuromusculoesqueléticas e relacionadas com o movimento } \\
\text { b730 - Funções da força muscular } \\
\text { b7300 - Força de músculos isolados e grupos musculares }\end{array}$ & $\begin{array}{l}1,8 \pm 0,7 \\
1,3 \pm 0,9 \\
2,3 \pm 0,6\end{array}$ & $\begin{array}{l}1,2 \pm 0,5 \\
0,6 \pm 0,7 \\
1,9 \pm 0,7\end{array}$ & $\begin{array}{l}0,0^{*} \\
0,0^{*} \\
0,0^{*}\end{array}$ & $\begin{array}{l}1,7 \pm 0,7 \\
1,2 \pm 0,9 \\
2,3 \pm 0,7\end{array}$ & $\begin{array}{l}1,2 \pm 0,5 \\
0,6 \pm 0,6 \\
1,8 \pm 0,7\end{array}$ & $\begin{array}{l}0,0^{*} \\
0,0^{*} \\
0,0^{*}\end{array}$ \\
\hline $\begin{array}{l}\text { s7 - Estruturas relacionadas com o movimento } \\
\text { s720 - Estrutura da região do ombro } \\
\text { s73001 - Articulações da região do cotovelo } \\
\text { s73002 - Músculos do braço } \\
\text { s7301 - Estrutura do antebraço } \\
\text { s73011 - Articulação do punho } \\
\text { s7302 - Estrutura da mão }\end{array}$ & $\begin{array}{l}1,2 \pm 0,9 \\
1,4 \pm 0,9 \\
1,4 \pm 0,9 \\
1,4 \pm 0,9 \\
1,4 \pm 0,9 \\
1,0 \pm 1,3 \\
0,7 \pm 1,2\end{array}$ & $\begin{array}{l}0,5 \pm 0,7 \\
0,6 \pm 0,8 \\
0,6 \pm 0,8 \\
0,6 \pm 0,8 \\
0,6 \pm 0,8 \\
0,4 \pm 0,8 \\
0,3 \pm 0,7\end{array}$ & $\begin{array}{l}0,0^{*} \\
0,0^{*} \\
0,0^{*} \\
0,0^{*} \\
0,0^{*} \\
0,0^{*} \\
0,0^{*}\end{array}$ & $\begin{array}{l}1,1 \pm 1,0 \\
1,4 \pm 1,0 \\
1,4 \pm 1,0 \\
1,4 \pm 1,0 \\
1,4 \pm 1,0 \\
1,0 \pm 1,3 \\
0,7 \pm 1,2\end{array}$ & $\begin{array}{l}0,5 \pm 0,6 \\
0,6 \pm 0,8 \\
0,6 \pm 0,8 \\
0,6 \pm 0,8 \\
0,6 \pm 0,8 \\
0,3 \pm 0,6 \\
0,2 \pm 0,6\end{array}$ & $\begin{array}{l}0,0^{*} \\
0,0^{*} \\
0,0^{*} \\
0,0^{*} \\
0,0^{*} \\
0,0^{*} \\
0,0^{*}\end{array}$ \\
\hline s73022 - Músculos da mão & $0,7 \pm 1,2$ & $0,3 \pm 0,7$ & $0,0^{*}$ & $0,7 \pm 1,2$ & $0,2 \pm 0,6$ & $0,0^{*}$ \\
\hline $\begin{array}{l}\text { d1 - Aprendizagem e aplicação de conhecimentos } \\
\text { d163 - Pensar }\end{array}$ & $2,2 \pm 0,7$ & $1,6 \pm 0,6$ & $0,0^{*}$ & $2,2 \pm 0,7$ & $1,5 \pm 0,6$ & $0,0^{*}$ \\
\hline $\begin{array}{l}\text { d2 - Tarefas e exigências gerais } \\
\text { d210 - Realizar uma única tarefa } \\
\text { d220 - Realizar tarefas múltiplas }\end{array}$ & $\begin{array}{l}1,9 \pm 0,9 \\
1,4 \pm 1,2 \\
2,5 \pm 1,0\end{array}$ & $\begin{array}{l}1,0 \pm 0,6 \\
0,6 \pm 0,6 \\
1,4 \pm 0,9\end{array}$ & $\begin{array}{l}0,0^{*} \\
0,0^{*} \\
0,0^{*}\end{array}$ & $\begin{array}{l}1,9 \pm 0,9 \\
1,3 \pm 1,2 \\
2,4 \pm 1,0\end{array}$ & $\begin{array}{l}0,9 \pm 0,6 \\
0,5 \pm 0,5 \\
1,4 \pm 0,9\end{array}$ & $\begin{array}{l}0,0^{*} \\
0,0^{*} \\
0,0^{*}\end{array}$ \\
\hline $\begin{array}{l}\text { d3 - Comunicação } \\
\text { d360 - Utilização de dispositivos e de técnicas de comunicação }\end{array}$ & $1,4 \pm 1,9$ & $0,3 \pm 1,1$ & $0,0^{*}$ & $1,2 \pm 1,9$ & $0,4 \pm 1,1$ & $0,0^{*}$ \\
\hline $\begin{array}{l}\text { d4 - Mobilidade } \\
\text { d420 - Autotransferências } \\
\text { d4401 - Agarrar } \\
\text { d450 - Andar } \\
\text { d455 - Deslocar-se } \\
\text { d4553 - Saltar } \\
\text { d465 - Deslocar-se utilizando algum tipo de equipamento } \\
\text { d470 - Utilização de transporte }\end{array}$ & $\begin{array}{l}2,2 \pm 1,4 \\
1,6 \pm 1,6 \\
0,7 \pm 1,2 \\
1,9 \pm 1,8 \\
1,9 \pm 1,8 \\
2,6 \pm 1,8 \\
1,9 \pm 1,9 \\
3,5 \pm 1,3\end{array}$ & $\begin{array}{l}1,1 \pm 1,1 \\
0,6 \pm 1,1 \\
0,3 \pm 0,7 \\
0,8 \pm 1,5 \\
0,8 \pm 1,5 \\
1,5 \pm 1,8 \\
0,8 \pm 1,5 \\
2,6 \pm 1,9\end{array}$ & $\begin{array}{l}0,0^{*} \\
0,0^{*} \\
0,0^{*} \\
0,0^{*} \\
0,0^{*} \\
0,0^{*} \\
0,0^{*} \\
0,0^{*}\end{array}$ & $\begin{array}{l}2,0 \pm 1,4 \\
1,7 \pm 1,6 \\
0,7 \pm 1,2 \\
1,9 \pm 1,8 \\
1,9 \pm 1,8 \\
2,6 \pm 1,7 \\
1,9 \pm 1,8 \\
3,5 \pm 1,3\end{array}$ & $\begin{array}{l}0,9 \pm 0,9 \\
0,4 \pm 0,9 \\
0,2 \pm 0,6 \\
0,7 \pm 1,3 \\
0,7 \pm 1,3 \\
1,3 \pm 1,7 \\
0,7 \pm 1,3 \\
2,4 \pm 1,9\end{array}$ & $\begin{array}{l}0,0^{*} \\
0,0^{*} \\
0,0^{*} \\
0,0^{*} \\
0,0^{*} \\
0,0^{*} \\
0,0^{*} \\
0,0^{*}\end{array}$ \\
\hline
\end{tabular}


Tabela 4 (continuação)

\begin{tabular}{|c|c|c|c|c|c|c|}
\hline & G1 & G2 & p & G3 & G4 & p \\
\hline d5 - Autocuidados & $2,0 \pm 1,1$ & $1,0 \pm 1,0$ & $0,0^{*}$ & $1,9 \pm 1,2$ & $0,9 \pm 0,9$ & $0,0^{*}$ \\
\hline d510 - Lavar-se & $3,0 \pm 1,7$ & $1,7 \pm 1,9$ & $0,0^{*}$ & $2,9 \pm 1,8$ & $1,6 \pm 1,9$ & $0,0^{*}$ \\
\hline d520 - Cuidar de partes do corpo & $1,8 \pm 2,0$ & $0,8 \pm 1,6$ & $0,0^{*}$ & $1,7 \pm 1,9$ & $0,7 \pm 1,5$ & $0,0^{*}$ \\
\hline d530 - Cuidados relacionados com os processos de excreção & $1,5 \pm 1,6$ & $0,5 \pm 1,1$ & $0,0^{*}$ & $1,5 \pm 1,6$ & $0,4 \pm 0,9$ & $0,0^{*}$ \\
\hline d540 - Vestir-se & $2,1 \pm 1,6$ & $0,9 \pm 1,5$ & $0,0^{*}$ & $2,0 \pm 1,7$ & $0,8 \pm 1,3$ & $0,0^{*}$ \\
\hline d550 - Comer & $0,6 \pm 0,9$ & $0,3 \pm 0,8$ & 0,1 & $0,7 \pm 1,0$ & $0,1 \pm 0,5$ & $0,0^{*}$ \\
\hline d5702 - Manter a própria saúde & $3,1 \pm 1,7$ & $2,1 \pm 2,0$ & $0,0^{*}$ & $3,0 \pm 1,7$ & $2,1 \pm 2,0$ & $0,0^{*}$ \\
\hline d6 - Vida doméstica & $3,1 \pm 1,2$ & $2,2 \pm 1,6$ & $0,0^{*}$ & $3,2 \pm 1,2$ & $1,9 \pm 1,5$ & $0,0^{*}$ \\
\hline d6200 - Comprar & $4,0 \pm 0,0$ & $2,9 \pm 1,8$ & $0,0^{*}$ & $3,9 \pm 0,5$ & $2,8 \pm 1,9$ & $0,0^{*}$ \\
\hline d630 - Preparar refeições & $3,5 \pm 1,3$ & $2,6 \pm 1,9$ & $0,0^{*}$ & $3,4 \pm 1,4$ & $2,6 \pm 1,9$ & $0,0^{*}$ \\
\hline d640 - Realizar as tarefas domésticas & $2,5 \pm 1,9$ & $1,5 \pm 1,9$ & $0,0^{*}$ & $2,7 \pm 1,9$ & $1,1 \pm 1,8$ & $0,0^{*}$ \\
\hline d6400 - Lavar e secar roupa & $2,9 \pm 1,8$ & $2,4 \pm 1,9$ & 0,2 & $3,2 \pm 1,7$ & $2,1 \pm 2,0$ & $0,0^{*}$ \\
\hline d650 - Cuidar dos objectos da casa & $2,5 \pm 1,9$ & $1,5 \pm 1,9$ & $0,0^{*}$ & $2,7 \pm 1,9$ & $1,1 \pm 1,8$ & $0,0^{*}$ \\
\hline \multicolumn{7}{|l|}{ d8 - Áreas principais da vida } \\
\hline d860 - Transacções econômicas básicas & $2,4 \pm 1,9$ & $0,9 \pm 1,7$ & $0,0^{*}$ & $2,3 \pm 1,9$ & $0,8 \pm 1,6$ & $0,0^{*}$ \\
\hline
\end{tabular}

G1, grupo 1 - baixo perfil na velocidade de alcance tarefas de atenção ( $<0,06 \mathrm{~m} / \mathrm{s}) ; \mathrm{G} 2$, grupo 2 - baixo perfil na velocidade de alcance tarefas de atenção ( $>0,06 \mathrm{~m} / \mathrm{s}) ; \mathrm{G} 3$, grupo 3 - baixo perfil na velocidade de alcance tarefas de memória $(<0,07 \mathrm{~m} / \mathrm{s}) ; \mathrm{G} 4$, grupo 4 - alto perfil na velocidade de alcance tarefas de memória ( $>0,07 \mathrm{~m} / \mathrm{s})$.

* $\mathrm{p}<0,05$, diferenças significativas entre os dois grupos (G1 e G2) do perfil de velocidade de alcance tarefas de atenção e entre os dois grupos (G3 e G4) do Perfil de Velocidade de Alcance com Memória (teste $t$-student para amostras independentes).

Considerando os resultados obtidos no AA e no $\mathrm{AM}$, os valores demonstram que o grupo de baixo perfil de velocidade de alcance (G1, G3) apresenta grande parte das categorias com um qualificador médio acima de 2 (moderadamente, gravemente ou completamente afetada). Permanecem acima de 3 as seguintes categorias: d470 - utilização de transporte $(3,5 \pm 1,3)$, d5 10 - lavar-se $(3.0 \pm 1,7)$, d5702 - manter a própria saúde $(3,1 \pm 1,7)$, d6 - vida doméstica $(3,1 \pm 1,2)$, d6200 - comprar $(4,0 \pm 0,0)$, d630 - preparar refeições $(3,5 \pm 1,3)$; especificamente no teste de AM mantém-se acima de um qualificador 3 a categoria d6400 - lavar e secar roupa $(3,2 \pm 1,7)$ Estes resultados não se mantêm no grupo de elevado perfil (G2, G4), não existindo nenhuma categoria neste grupo com o qualificador 3 . Apesar disso, houve algumas que se mantiveram acima de 2: d470 - utilização de transporte $(2,6 \pm 1,9)$, d5702 - manter a própria saúde $(2,1 \pm 2.0)$, d6200 - comprar $(2.9 \pm 1,8), \mathrm{d} 630$ - preparar refeições $(2,6 \pm 1,9)$ e d6400 - lavar e secar roupa $(2,4 \pm 2,0)$. Especificamente no AM, mantém-se acima do 2 a categoria d6 - vida doméstica $(2.2 \pm 1,6)$ (Figuras 2 e 3).

Os grupos de baixo perfil funcional detetados em ambos os testes (alcance em tarefas de atenção e alcance em tarefas de memória) não evidenciaram diferenças estatisticamente significativas nos qualificadores de $1^{\mathrm{O}}$ nível $(\mathrm{p}>0,05)$ (Figura 4). Através da análise dos códigos da CIF classificados com diferentes níveis de qualificadores nos grupos de baixo perfil funcional na velocidade de alcance, é percetível que: a componente mais afetada é a d6. Vida doméstica (qualificadores CIF 3-4); seguindo-se as categorias d1. Aprendizagem e aplicação de conhecimento, d4. Mobilidade, d5. Autocuidados e d8. Áreas significativas da vida (educação, trabalho, etc.) (qualificadores CIF 2-3). Num nível ligeiro a moderado (qualificadores 1-2) estão as categorias s7. Estruturas relacionadas com o movimento, b1. Funções mentais, b6. Funções genitourinárias, b7. Funções neuromúsculo-esqueléticas, d2. Tarefas e exigências gerais e d3. Comunicação. Por outro lado, a categoria menos afetada é b5. Funções defecação (qualificadores 0-1).

\section{DISCUSSÃO}

Em síntese, os resultados mostraram diferenças significativas nos utentes com baixo e elevado perfil de velocidade de alcance em tarefas de atenção (valores $<0.06 \mathrm{~m} / \mathrm{s}$ ) e memória (valores $<0.07 \mathrm{~m} / \mathrm{s}$ ) ajudando a detectar as seguintes áreas prioritárias de intervenção em idosos institucionalizados: d6. Vida doméstica (qualificadores CIF 3-4), d1. Aprendizagem e aplicação de conhecimento, d4. Mobilidade, d5. Autocuidados e d8. Áreas significativas da vida (educação, trabalho, etc.). Assim, os resultados apresentados no presente estudo permitem concluir que o Teste do Alcance sugerido parece ser promissor no processo de avaliação da capacidade funcional na população idosa, sendo determinante para discriminar áreas de reabilitação de interesse na geriatria. 


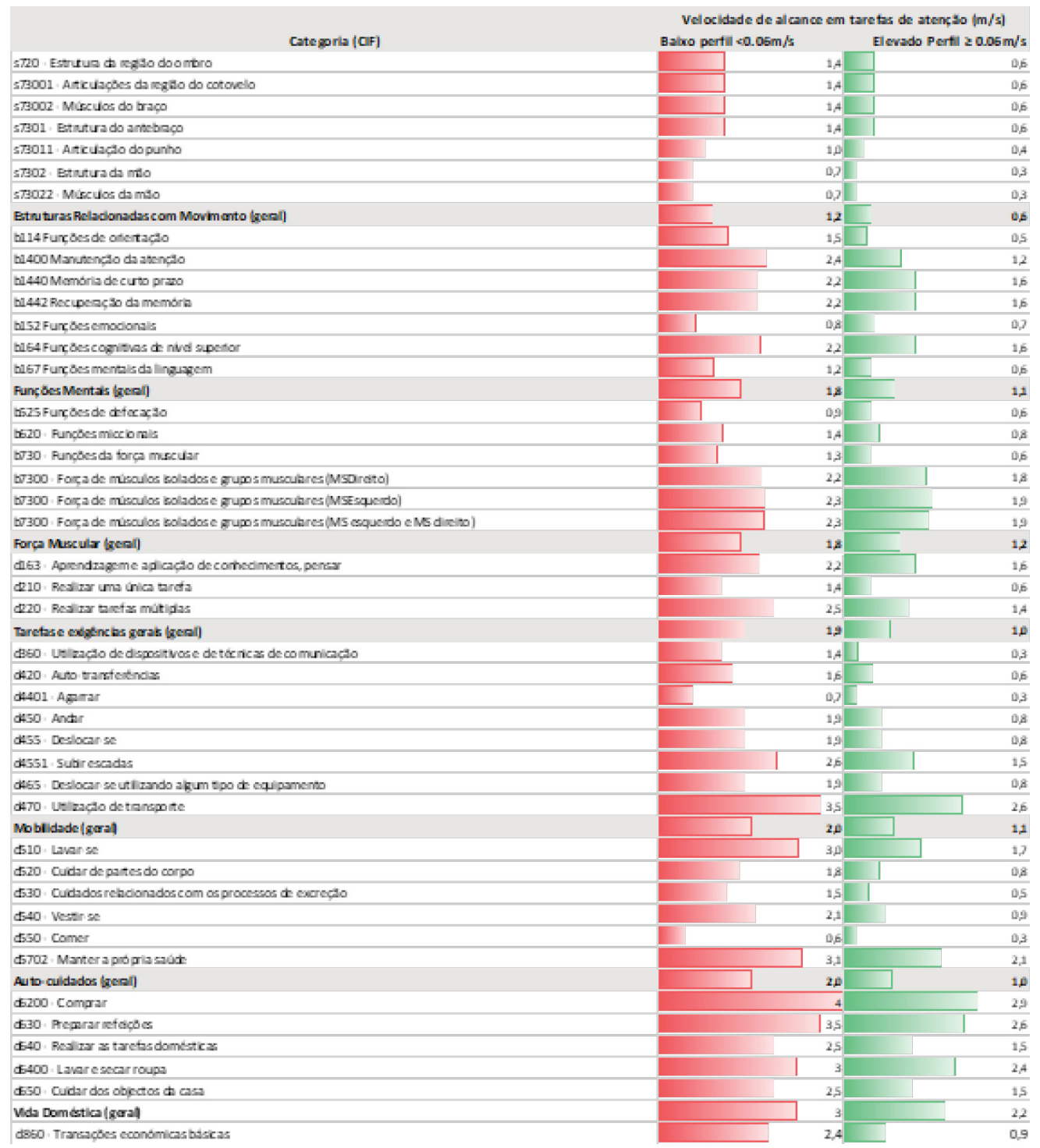

Figura 2. Perfil funcional dos grupos de baixo e elevado perfil de velocidade de alcance em tarefas de atenção (AA)

Desta forma, este estudo foi um contributo para a validação de indicadores funcionais dedicados à avaliação multidimensional (psicomotora) do membro superior. Os poucos estudos que apresentaram indicadores para o membro superior associados à funcionalidade estão especialmente dedicados à avaliação da função motora fina, como é o caso do estudo conduzido por Federici et al. [35] desenvolvido com idosos internados numa unidade de enfermaria e com foco na avaliação e treino das funções da pinça e do controlo motor fino da mão. A importância da reeducação da mão é confirmada no estudo de Rosa et al. [20] como um aspeto determinante para a saúde geral do idoso institucionalizado, tendo sido pouco explorada a função do alcance neste contexto.
Para idosos com potencial de marcha, os testes de velocidade de marcha estão amplamente estudados e demonstram estar associados à fragilidade funcional do idoso [36]. Frequentemente, em estudos relacionados com a identificação de estados frágeis na pessoa idosa, são identificados os domínios da mobilidade, da independência em AVD e nos autocuidados, como significativos indicadores de fragilidade [37-39]. Os resultados do presente estudo parecem assim indicar estarem relacionados com os perfis de fragilidade dos idosos, pelo que futuros estudos sobre a Velocidade do Alcance em Tarefas de Atenção e em Tarefas de Memória devem procurar relacionar os seus valores com Índices de Fragilidade. 


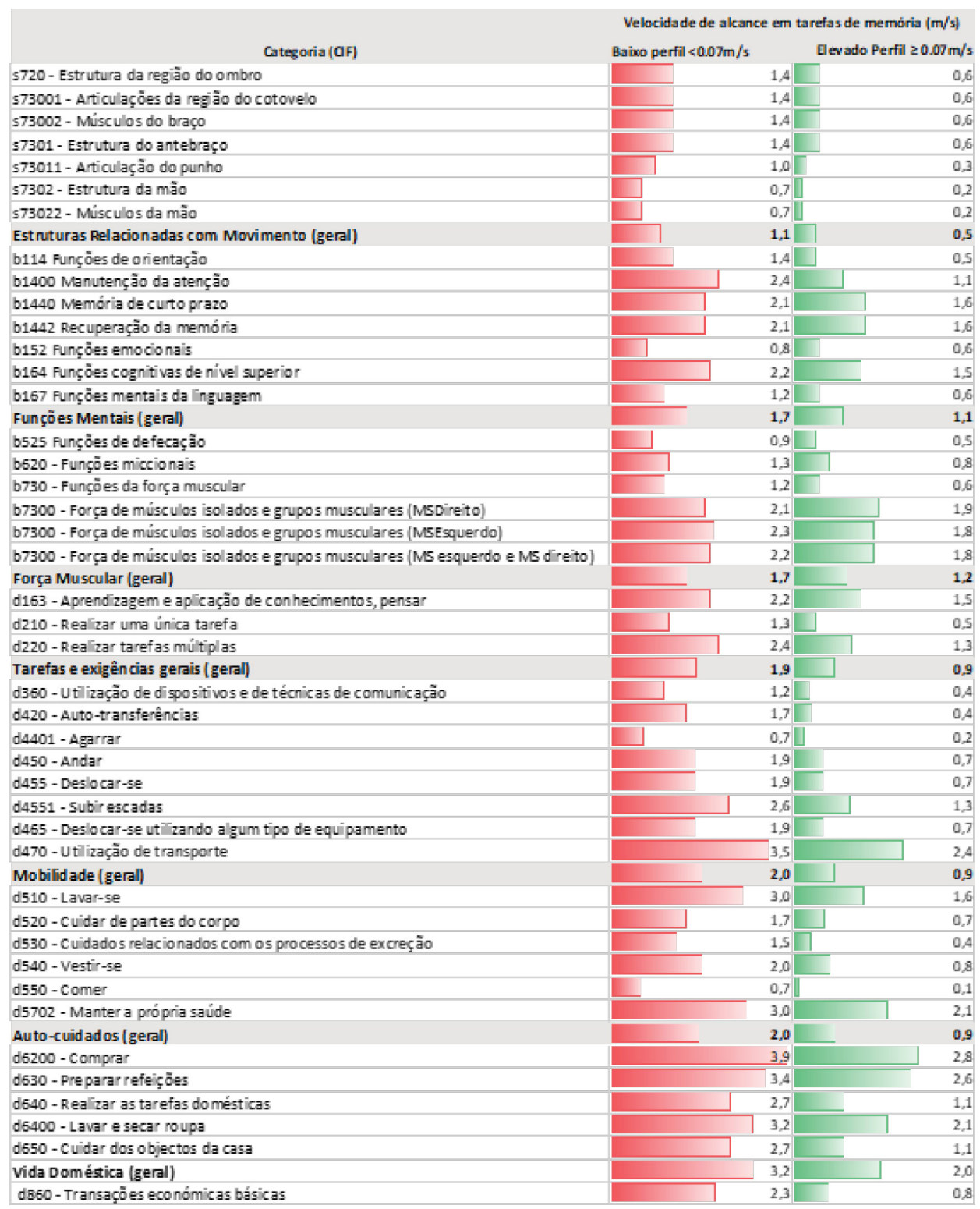

Figura 3. Perfil funcional dos grupos de baixo e elevado perfil de velocidade de alcance em tarefas de memória.

Grupos de Balxo Perfll Funclonal

57. Estruturas relacl onadas com o movi merto

b1.Funçes mentals

b5. Funç̄oses defecaçăo

b6. Funçbes Genitourinárlas

b7. Funçese neuromusculo-esqueléticas

d1. Aprendil zagem e aplicaça de conheci mento

d2. Tarefas e Exigenclas Gerals

d3. Comunicaça

d4. Mobilidade

d5. Auto-culdados

d5. Vida domestica

d8. Areas slgnificativas da vida (educaçă trabalho, etc.)

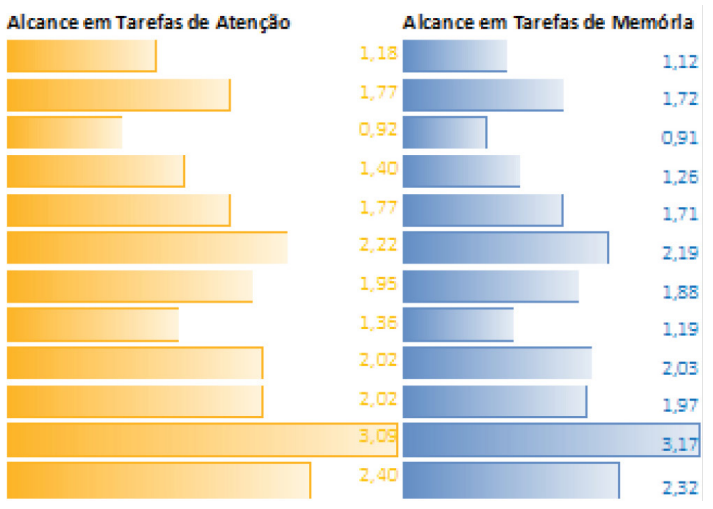

Figura 4. Comparação de qualificadores de 1ำ nível da Classificação Internacional de Funcionalidade entre grupos de baixo perfil para os testes de alcance em tarefas de atenção e para os testes de alcance em tarefas de memória. 
A implementação das regras de linking para a CIF são uma ferramenta muito útil na validação clínica do teste proposto, ajudando a compreender a sua habilidade na distinção de perfis funcionais [40]. Assim, os profissionais que possam ter interesse em implementar os testes de alcance (em tarefas de atenção e memória) na prática clínica ou investigação em idosos institucionalizados, podem facilmente reproduzir a metodologia sugerida, tendo por base a listagem de códigos de CIF sugerida. Desta forma, evitam-se limitações de reprodução metodológica, muitas vezes causada por inexistência de instrumentos de avaliação culturalmente adaptados ou não traduzidos para a língua oficial onde os estudos decorrem.

As atividades instrumentais estão predominantemente afetadas a um nível moderado-severo nos grupos de baixo perfil. Estes dados podem ser explicados pela natureza da nossa população, tratando-se de indivíduos institucionalizados. A população institucionalizada diariamente não é requisitada a desempenhar grande parte das atividades instrumentais e logicamente é natural que o seu desempenho neste tipo de tarefas seja limitado [41]. Para além disso, os instrumentos usados basearam-se em respostas dadas pelos idosos, sendo que a sua perceção de autonomia possa estar influenciada pelo processo de institucionalização, tal como confirmado pelo estudo de Jeon et al. [42]. Neste estudo, os autores concluem que a perceção de autonomia pode estar influenciada por alguma diversidade de fatores como a prática de atividade física, a idade avançada, a morte de alguém próximo, a dor e o nível de suporte providenciado pela própria instituição [42]. Assim, futuras investigações no contexto específico de idosos institucionalizados, devem incluir a avaliação deste conjunto de fatores.

Outro dado de interesse do presente estudo indica que o perfil funcional identificado pelo teste de Alcance em Tarefas de Atenção e pelo Teste de Alcance em Tarefas de Memória não apresentou dados estatisticamente diferentes. A metodologia usada na implementação do teste pode ter tido Influência nestes resultados, pois Teste de Atenção foi sempre realizado em primeiro lugar, numa lógica de progressão de dificuldade e só posteriormente foi solicitado o teste de alcance em tarefas de memória. Realizando primeiro o Teste de Atenção, existe alguma probabilidade de os participantes terem aprendido e retido informação, podendo ser um motivo para o aumento do desempenho na Tarefa de Memória. Contudo, um estudo anterior realizado por Walter et al. [43] sugere que esta capacidade de transferir competências na realização de uma tarefa para a outra está inversamente relacionada com a idade. Em futuros estudos, a implementação dos testes de Atenção e Memória deverá seguir uma ordem aleatória/alternada entre os si com a finalidade de verificar a influencia da metodologia nos valores do teste, podendo ainda considerar o fator idade como possível influenciador dos resultados.

O perfil funcional identificado pelo Teste de Alcance em Tarefas de Atenção foi idêntico ao perfil funcional identificado pelo Teste de Alcance em Tarefas de Memória ( $p>0.05)$. Limitações no desenho do estudo podem ter dificultado a análise da especificidade dos testes para identificar diferentes perfis funcionais. Uma das principais limitações poderá estar relacionada com o facto de os dois testes terem sido implementados ao mesmo grupo de pessoas, com características cognitivas, motoras e funcionais bastante dispersas. Esta diversidade dificultou a análise de perfis específicos para cada um dos testes. Por exemplo, para verificar a especificidade do Teste de Alcance em Tarefas de Memória, seria interessante testar em populações com perfis cognitivos específicos, nomeadamente numa população com demência, onde as questões relacionadas com as dificuldades da memória são comuns aos indivíduos. Para verificar a especificidade do teste de alcance durante as tarefas de atenção, futuros estudos poderão alargar para a comparação entre amostras de idosos que vivem na comunidade, considerando que as expectativas do seu nível de independência e autonomia funcional serão muito superiores às da população estudada [44]. Poderá ainda ser importante considerar estudos específicos em amostras de idosos institucionalizados com diferentes níveis de preservação na atenção seletiva. De uma forma geral, pode-se assim concluir que este estudo foi um importante contributo para validar a velocidade de alcance como um indicador funcional na população geriátrica, possibilitando a avaliação funcional em idosos não ambulantes.

Em conclusão, os resultados mostraram diferenças significativas nos utentes com baixo e elevado perfil de velocidade de alcance em tarefas de atenção e memória ajudando a detetar as seguintes áreas prioritárias de intervenção em idosos institucionalizados: d6. Vida doméstica (qualificadores CIF 3-4), d1. Aprendizagem e aplicação de conhecimento, d4. Mobilidade, d5. Autocuidados e d8. Áreas significativas da vida (educação, trabalho, etc.).

\section{NOTAS}

\section{Apoio financeiro}

Este estudo não recebeu apoio financeiro de fontes externas. 
Declaração de conflito de interesses

Os autores declaram não haver conflitos de interesses relevantes ao conteúdo deste estudo.

Contribuições dos autores

Todos os autores fizeram contribuições substanciais para concepção, ou delineamento, ou aquisição, ou análise ou inter- pretação de dados; e redação do trabalho ou revisão crítica; e aprovação final da versão para publicação.

Disponibilidade dos dados e responsabilidade pelos resultados

Todos os autores declaram ter tido total acesso aos dados obtidos e assumem completa responsabilidade pela integridade destes resultados.

\section{REFERÊNCIAS}

1. World Health Oganisation. Global health and ageing. Geneva: WHO; 2015.

2. Ferreira OGL, Maciel SC, Costa SMG, Silva AO, Moreira MASP. Envelhecimento ativo e sua relação com a independência funcional. Texto Context - Enferm. 2012;21(3):513-8. https://doi.org/10.1590/S010407072012000300004

3. Imaginário C, Machado $\mathrm{P}$, Antunes $\mathrm{C}$, Martins T. Perfil funcional de los ancianos institucionalizados en residencias: estudio piloto. Gerokomos. 2018;29(2):59-64.

4. Zulkowski K, Kindsfater D. Examination of care-planning needs for elderly newly admitted to an acute care setting. Ostomy Wound Manage. 2000;46(1):32-8.

5. Lauretani F, Maggio M, Ticinesi A, Tana C, Prati B, Gionti L, Nouvenne A, Meschi T. Muscle weakness, cognitive impairment and their interaction on altered balance in elderly outpatients: results from the TRIP observational study. Clin Interv Aging. 2018;13:1437-43. https://doi.org/10.2147/CIA.S165085

6. Mahoney FI, Barthel DW. Functional evaluation: The Barthel Index. Md State Med J. 1965;14:61-5. https://doi. org/10.1037/t02366-000

7. Lawton MP, Moss M, Fulcomer M, Kleban MH. A research and service oriented multilevel assessment instrument. J Gerontol. 1982;37(1):91-9. https://doi.org/10.1093/geronj/37.1.91

8. Hutchings L, Fox R, Chesser T. Proximal femoral fractures in the elderly: How are we measuring outcome? Injury. 2011;42(11):1205-13. https://doi.org/10.1016/j.injury.2010.12.016

9. Iconaru EI, Ciucurel MM, Georgescu L, Ciucurel C. Hand grip strength as a physical biomarker of aging from the perspective of a Fibonacci mathematical modeling. BMC Geriatr. 2018;18:296-304. https://doi.org/10.1186/ s12877-018-0991-0

10. Novo A, Preto L, Mendes E. A força de preensão manual como indicador da capacidade funcional em idosos. 1ํ Congresso de Cuidados Continuados; 28-29 set. 2012; Bragança, Portugal. Bragança: IPB; 2012.

11. Berg K, Wood-Dauphine S, Williams JI, Gayton D. Measuring balance in the elderly: preliminary development of an instrument. Physiother Canada. 1989;41(6):304-11. https://doi.org/10.3138/ptc.41.6.304

12. Berg K, Wood-Dauphinee S, Williams JI. The Balance Scale: reliability assessment with elderly residents and patients with an acute stroke. Scand J Rehabil Med. 1995;27(1):27-36.

13. Rodrigues RMC, Silva CFR, Loureiro LMJ, Silva SMDT, Crespo SSS, Azeredo ZAS. Os muito idosos: avaliação funcional multidimensional. Rev Enf Ref. 2015;(5):65-74. https://doi.org/10.12707/RIV14040

14. Alcazar J, Rodriguez-Lopez C, Ara I, Alfaro-Acha A, Mañas-Bote A, Guadalupe-Grau A, García-García FJ, Alegre LM. The force-velocity relationship in older people: reliability and validity of a systematic procedure. Int J Sport Med. 2017;38(14):1097-1104. https://doi.org/10.1055/s-0043-119880

15. Wolf SL, Catlin PA, Gage K, Gurucharri K, Robertson R, Stephen K. Establishing the reliability and validity of measurements of walking time using the emory functional ambulation profile. Phys Ther. 1999;79(12):1122-33.

16. Alencar MA, Dias JMD, Figueiredo LC, Dias RC. Força de preensão palmar em idosos com demência: estudo da confiabilidade. Rev Bras Fisioter. 2012;16(6):510-4. https://doi.org/10.1590/S1413-35552012005000059

17. Osoba MY, Rao AK, Agrawal SK, Lalwani AK. Balance and gait in the elderly: a contemporary review. Laryngoscope Investig Otolaryngol. 2019;4(1):143-53. https://doi.org/10.1002/lio2.252 
18. Umakalyani K, Senthil Kumar M. An observational study of gait speed in elderly participants attending outpatient clinic geriatric department, Madras Medical College, Chennai. J Evol Med Dent Sci. 2018;7(11):1356-9. https://doi. org/10.14260/jemds/2018/308

19. Rosa M, Amado S, Morouço P, Faria P, Batista R, Silva D. AGILidades. 604143/42PT; 2018.

20. Rosa MDD, Pires M, Ferreira L, Canaverde MJ, Martins N, Guardado J. Sensory-motor extremity function in institutionalised eldelry people: role of emotional and cognitive status. Thomson Reuteurs, Ageing Congress; 2019.

21. Pimentel AMF. Função cognitiva e fatores associados numa amostra representativa de idosos Portugueses na comunidade: resultados do Projeto Nutrition UP 65 [dissertação]. Porto: Universidade do Porto; 2017.

22. Adedoyin RA, Ogundapo FA, Mbada CE, Adekanla BA, Johnson OE, Onigbinde TA, Emechete AAI. Reference values for handgrip strength among healthy adults in Nigeria. Hong Kong Physiother J. 2009;27(1):21-9. https://doi. org/10.1016/S1013-7025[10]70005-1

23. Folstein MF, Folstein SE, McHugh PR. Mini-mental state: a grading the cognitive state of patiens for the clinician. J Psychiatr Res. 1975;12(3):189-98. https://doi.org/10.1016/0022-3956[75]90026-6

24. Brucki SMD, Mansur LL, Carthery-Goulart MT, Nitrini R. Formal education, health literacy and mini-mental state examination. Dement Neuropsychol. 2011;5(1):26-30. https://doi.org/10.1590/S1980-57642011DN05010005

25. Veríssimo MT. Avaliação diagnóstica dos síndromes demenciais: escala de depressão geriátrica. Porto: Universida do Porto; 1988.

26. Sequeira C. Cuidar de idosos dependentes. Coimbra: Quarteto Editora; 2007.

27. Dubois B, Slachevsky A, Litvan I, Pillon B. The FAB: a frontal assessment battery at bedside. Neurology. 2000;55(11):1621-6. https://doi.org/10.1212/WNL.55.11.1621

28. Fugl-Meyer AR, Jääskö L, Leyman I, Olsson S, Steglind S. The post-stroke hemiplegic patient. 1. a method for evaluation of physical performance. Scand J Rehabil Med. 1975;7(1):13-31.

29. Lawton MP, Brody EM. Assessment of older people: self-maintaining and instrumental activities of daily living. Gerontologist. 1969;9(3):179-86. https://doi.org/10.1093/geront/9.3_Part_1.179

30. Tomandl J, Book S, Gotthardt S, Heinmueller S, Graessel E, Freiberger E, Kuehlein T, Hueber S, Hoyer S. Laying the foundation for a core set of the International classification of functioning, disability and health for community-dwelling adults aged 75 years and above in general practice: a study protocol. BMJ Open. 2018;8(8): e024274. https://doi.org/10.1136/bmjopen-2018-024274

31. Cieza A, Geyh S, Chatterji S, Kostanjsek N, Üstün B, Stucki G. ICF linking rules: an update based on lessons learned. J Rehabil Med. 2005;37(4):212-8. https://doi.org/10.1080/16501970510040263

32. Cieza A, Fayed N, Bickenbach J, Prodinger B. Refinements of the ICF linking rules to strengthen their potential for establishing comparability of health information. Disabil Rehabil. 2019;41(5):574-83. https://doi.org/10.3109/09638288. 2016.1145258

33. Cornelis E, Gorus E, Beyer I, Bautmans I, De Vriendt P. Early diagnosis of mild cognitive impairment and mild dementia through basic and instrumental activities of daily living: development of a new evaluation tool. PLoS Med. 2017;14(3):e1002250. https://doi.org/10.1371/journal.pmed.1002250

34. Velstra I-M. Advanced insights in upper limb function of individuals with cervical spinal cord injury. Enschede: Amsterdam; 2015.

35. Federici A, Conteduca BR, Lucertini F, Dell'Anna S, Marini CF, Vetri M. Effect of a psychomotor training program on hand function in nursing home residents: a pilot study. J Phys Educ Sport. 2018;18(2):627-31.

36. Pamoukdjian F, Paillaud E, Zelek L, Laurent M, Lévy V, Landre T, Sebbane G. Measurement of gait speed in older adults to identify complications associated with frailty: a systematic review. J Geriatr Oncol. 2015;6(6):484-96. https://doi.org/10.1016/j.jgo.2015.08.006

37. Kannegieter LM, Tap L, Oudshoorn C, van Bruchem-Visser RL, Mattace-Raso FUS. Mobility and handgrip strength but not aortic stiffness are associated with frailty in the elderly. J Gerontol Geriatr [Internet]. 2016;64:2-8.

38. Nourhashémi F, Andrieu S, Gillette-Guyonnet S, Vellas B, Albarède JL, Grandjean H. Instrumental activities of daily living as a potential marker of frailty: a study of 7364 community-dwelling elderly women (the EPIDOS Study). J Gerontol A Biol Sci Med Sci. 2001;56(7):M448-53. https://doi.org/10.1093/gerona/56.7.M448 
39. Jakobsson U, Karlsson S. Predicting mortality with the ADL-staircase in frail elderly. Phys Occup Ther Geriatr. 2011;29(2):136-47. https://doi.org/10.3109/02703181.2010.538491

40. de Moura L, dos Santos WR, Castro SS, Ito E, da Luz e Silva DC, Yokota RTC, Abaakouk Z, Corrêa Filho HR, Gomes Pérez MA, Fellinghauer CS, Sabariego C. Applying the ICF linking rules to compare population-based data from different sources: an exemplary analysis of tools used to collect information on disability. Disabil Rehabil. 2019;41(5):601-12. https://doi.org/10.1080/09638288.2017.1370734

41. Veloso LSG, Freire BHF, Veloso JAP, Gouveia MLA, Colella TLA, Moreira MASP. Analysis of the functional performance of self-institutionalized elderly people. Fundam Care. 2018;10(4):1176-81. https://doi.org/10.9789/ 2175-5361.2018.v10i4.1176-1181

42. Jeon Y-H, Tudball J, Nelson K. How do residents of aged care homes perceive physical activity and functional independence? A qualitative study. Health Soc Care Community. 2019;27(5):1321-32. https://doi.org/10.1111/ hsc. 12777

43. Walter CS, Hengge CR, Lindauer BE, Schaefer SY. Declines in motor transfer following upper extremity task-specific training in older adults. Exp Gerontol. 2019;116:14-9. https://doi.org/10.1016/j.exger.2018.12.012

44. Mugo JW, Onywera VO, Waudo JN, Otieno OG. Functionality of elderly persons: a comparison between institutionalized and non - institutionalized elderly persons in Nairobi City County, in Kenya. World J Public Heal. 2018;3(1):9-15. https://doi.org/10.11648/j.wjph.20180301.12 